\title{
SAT-based Summarization for Boolean Programs
}

\author{
Gérard Basler^, Daniel Kroening, and Georg Weissenbacher ${ }^{\star \star}$ \\ Computer Systems Institute, ETH Zurich, 8092 Zurich, Switzerland \\ \{firstname.lastname\}@inf.ethz.ch
}

\begin{abstract}
Boolean programs are frequently used to model abstractions of software programs. They have the advantage that reachability properties are decidable, despite the fact that their stack is not bounded. The enabling technique is summarization of procedure calls. Most model checking tools for Boolean programs use BDDs to represent these summaries, allowing for an efficient fix-point detection. However, BDDs are highly sensitive to the number of state variables. We present an approach to over-approximate summaries using Bounded Model Checking. Our technique is based on a SAT solver and requires only few calls to a QBF solver for fix-point detection. We present benchmarks that show that our implementation is able handle a larger number of variables than BDD-based algorithms on some examples.
\end{abstract}

\section{Introduction}

Boolean programs [1] are frequently used to model software programs. They provide the usual control-flow constructs of an imperative language such as C, but variables are exclusively of Boolean type. The use of Boolean programs as an abstract model has been promoted by the success of the Slam project [2]. SLAM verifies control-flow dominated properties of Windows device drivers by abstracting an ANSI-C program into a Boolean program. The abstract model of the original program is obtained by means of Predicate Abstraction [3]. It contains the same procedures and control flow as the original program, and thus, Boolean programs are a natural formalization. The Boolean variables are used to keep track of predicates over the variables of the original program.

The main advantage of Boolean programs over finite-state transition systems is that their stack allows a precise representation of the behavior of procedure calls, including procedure-local variables and (possibly unbounded) recursive calls. Nevertheless, reachability properties for Boolean programs are decidable, despite of the fact that the stack is not depth-bounded [4]: Procedures can access and modify only the topmost element of the stack. Therefore, summarizing the procedures prevents a re-evaluation of a call if the same calling context has already been considered before [5].

\footnotetext{
* Supported by the Swiss National Science Foundation.

** Supported by a Microsoft Research European PhD scholarship.
} 
Most of the existing model checkers compute summaries incrementally at each call site. The reachable states are determined by means of a saturation procedure, which computes and adds summaries until no new states are discovered. Instances of such model checkers are BEBOP [1], which is shipped with SLAM, and Moped [6]. Both tools use a symbolic representation of states and summaries based on Binary Decision Diagrams (BDDs) [7]. BDDs are a canonical representation of formulas and can be compared efficiently, thus enabling the detection of previously explored portions of the state space.

However, in case of a large number of state variables, the BDDs become unmanageably large. Approaches based on SAT-solvers (e.g. [8]) are less sensitive to the number of variables. However, they suffer from the fact that fix-point detection requires a decision procedure for quantified Boolean formulas (QBF), making it significantly harder than with BDDs.

We propose a SAT-based summarization technique that reduces the required number of QBF calls significantly. We propose to exploit the following observation about Boolean programs generated by tools such as SLAM: They are shallow. Formally, this means that the length of the shortest path from an initial state to any reachable valuation of global and local variables is bounded by a small constant. This bound is called sequential depth. Programs written in a general purpose programming language such as ANSI-C have this property only in few cases. A single loop with an integer counter variable may result in a Kripke structure with loop-free paths as long as $2^{32}$.

Obviously, a Boolean program may just as well have a sequential depth that is exponential in the number of Boolean variables it contains. However, such a program must encode the equivalent of a binary counter using a propositional relation over the Boolean variables. Such models are typically not generated by any of the program analysis tools that we experimented with, as the whole point of an abstract Boolean model is to remove such counter variables.

In order to replace the expensive fixed-point detection, we propose to generate a universal summary, which encodes all possible traces through the procedure up to its sequential depth, starting from an uninitialized calling context. A universal summary is an over-approximation of the fix-point that incremental summarization would yield.

Computing the exact sequential depth is as hard as model checking. Therefore, we use the reachability recurrence diameter, an over-approximation that is commonly used in bounded model checking (BMC) and can be computed by means of a SAT solver.

Related Work Boolean programs have been introduced as a formalism to represent abstract models generated by Slam [9]. The success of the SLAM project motivated many researchers to work on even faster model checking algorithms for Boolean programs. However, the formalism is equally expressive as Pushdown systems, which have been studied long before Slam was presented.

Büchi proved already in 1964 that the set of reachable states of a pushdown system (represented by a string rewriting system) can be expressed in terms of 
a regular language [4]. Sharir and Pnueli present a dataflow analysis algorithm based on iterative fix-point detection that makes use of summarization [5]. Ball and Rajamani's model checker BEBOP is based on this work, but uses BDDs to represent states symbolically [1]. Finkel et al. present an automata-based saturation algorithm that constructs the regular set representing the reachable states of a pushdown system [10]. Esparza presents an optimized version of Finkel's algorithm [6], and Schwoon improves this approach using a BDD-based symbolic representation of pushdown systems [11]. Lal and Reps present a graphtheoretic approach for model checking (weighted) pushdown systems [12]. None of the approaches listed above is based on satisfiability solving techniques.

Boujjani and Esparza survey approaches that use rewriting to solve the reachability problem for sequential as well as for concurrent pushdown systems [13]. Rewriting-based reachability analysis of concurrent pushdown system is also covered by [14] and [15]. However, the reachability problem for concurrent pushdown is undecidable [16]. Various approaches have been considered to overcome this problem (e.g., [17-19] and [14]). We omit a discussion of this work, since our approach does not target concurrent Boolean programs.

Leino's model checker Dizzy [20] uses SAT-based symbolic simulation. However, the fix-point detection is still done by computing BDDs representing the set of reachable states.

Kroening presents a SAT-based model checker called BopPo for concurrent pushdown systems without recursive procedures [8]. This work comes closest to our approach, since BopPo uses a SAT-solver for symbolic simulation and a QBF-solver for fix-point detection. Procedure calls can be simulated by dynamic threads; However, in the presence of threads, Boppo computes an overapproximation of the set of reachable states. Furthermore, Boppo does not generate summaries for procedures.

Our approach is different from the model checking algorithms listed above, since we compute an over-approximation of the summaries of a Boolean program, instead of computing the least fix-point of this set. To compute this overapproximation, we use Bounded Model Checking (BMC).

BMC was introduced by Biere [21] as a SAT-based alternative to finite-state model checking algorithms that use Binary Decision Diagrams (BDDs) [7]. BMC searches for counterexamples to a property with a bounded length $k$, which is increased iteratively. The approach is complete if $k$ exceeds the completeness threshold, a concept that was introduced in [22]. The recurrence diameter is an over-approximation of the completeness threshold for reachability properties in of finite-state transition systems, which can be computed using a SAT solver [22].

Contribution and Outline We present background on Boolean programs and BMC in Sec. 2. In Section 3, we present a SAT-based model checking algorithm computes a set of summary edges for each procedure and finds the least fixpoint of these sets using a QBF solver. This work is based on the algorithm presented in [1]. However, to the best of our knowledge, our tool is the first one that implements summarization using SAT and QBF. 
In Section 4, we introduce the notion of a universal summary, which encodes the unwinding of all possible execution traces of a procedure up to its reachability recurrence diameter. We explain how the use of universal summaries can significantly reduce the number of calls to the QBF solver.

We have implemented these algorithms for model checking Boolean programs and provide experimental results comparing this implementation to a conventional BDD-based algorithm in Section 5.

\section{Background}

The construction of universal summaries is based on an over-approximation of what we call the sequential depth of a procedure. We borrow this idea from Bounded Model Checking.

\subsection{Bounded Model Checking}

$\mathrm{BMC}$ is a method for finding logical errors in finite-state transition systems. It is widely regarded as a complementary technique to symbolic BDD-based model checking, and frequently used in the hardware industry; see [23] for a survey of experiments with BMC conducted in industry.

Definition 1 (Finite-State Transition System). A Finite-State Transition System $\mathcal{M}=\left\langle S, T, s_{0}\right\rangle$ is defined by a finite set of states $S$, a transition relation $T \subseteq S \times S$, and an initial state $s_{0} \in S$.

Given a finite-state transition system $\mathcal{M}$, an LTL property $\varphi$, and a natural number $k$, a BMC procedure decides whether there exists a computation in $\mathcal{M}$ of length $k$ or less that violates $\varphi$. SAT-based BMC is performed by generating a propositional formula, which is satisfiable if and only if such a path exists. We write $M \models_{k} \varphi$ if all sequences of transitions up to length $k$ satisfy $\varphi$.

In practice, the application of BMC is typically restricted to the refutation of safety properties, and is conducted in an iterative process: Starting with a small initial value of $k, k$ is incremented until 1) an error is found, or 2) the problem becomes intractable due to the complexity of solving the corresponding SAT instance.

Bounded Model Checking is complete iff $k$ reaches a completeness threshold $\mathcal{C T}$, which indicates there exists no path in $M$ that violates $\phi$.

Definition 2 (Completeness Threshold [22]). A completeness threshold of a transition system $\mathcal{M}$ with respect to a property $\varphi$ is any natural number $\mathcal{C} \mathcal{T}$ such that, given that the property $\varphi$ is not violated by any deduction of length up to $\mathcal{C} \mathcal{T}$, then it cannot be violated at all, i.e.,

$$
M \vDash \mathcal{C} \mathcal{T} \phi \Longrightarrow M \models \phi
$$

holds. 
The reachability diameter of a finite-state transition system $\mathcal{M}$ is a completeness threshold for reachability properties of the form $\mathbf{G} p$ :

Definition 3 (Reachability Diameter [22]). The Reachability Diameter $r d(M)$ of a finite-state transition system $M$ is the minimal number of steps required for reaching all reachable states.

The reachability diameter corresponds to our notion of the sequential depth.

Clearly, if $M \models \varphi$, then the smallest $\mathcal{C} \mathcal{T}$ is 0 , and otherwise it is equal to the length of the shortest counterexample. This implies that finding the smallest $\mathcal{C T}$ is at least as hard as checking $M \models \varphi$. Consequently, we concentrate on computing an over-approximation of the smallest $\mathcal{C} \mathcal{T}$.

Definition 4 (Reachability Recurrence Diameter [22]). The Reachability Recurrence Diameter with respect to a model $\mathcal{M}=\left\langle S, T, s_{0}\right\rangle$ is the longest loopfree path in $\mathcal{M}$ starting from the initial state $s_{0}$ :

$$
\operatorname{rrd}(\mathcal{M}) \stackrel{\text { def }}{=} \max \left\{i \mid \exists s_{1} \ldots s_{i} . \bigwedge_{j=0}^{i-1} T\left(s_{j}, s_{j+1}\right) \wedge \bigwedge_{j=0}^{i-1} \bigwedge_{k=j+1}^{i} s_{j} \neq s_{k}\right\}
$$

The Reachability Diameter and the Reachability Recurrence Diameter are only defined for models with a finite state space. However, Boolean programs do not adhere to this restriction, since they contain procedures with call-by-value parameter passing and recursion.

\subsection{Semantics of Boolean Programs}

We define Boolean programs and their semantics in terms of the control-flow graph of a program. A Boolean program consists of a set of procedures, each of which is represented by its control flow graph (CFG).

As usual, a control flow graph is a directed graph with nodes corresponding to program locations. Without loss of generality, we assume that each procedure has exactly one entry node and one exit node.

Each edge $\left\langle n_{1}, n_{2}\right\rangle$ corresponds to a transition function $\left\langle n_{1}, \Omega_{1}\right\rangle \rightarrow\left\langle n_{2}, \Omega_{2}\right\rangle$ that relates the values of the variables in scope before the transition $\left(\Omega_{1}\right)$ to those after the transition $\left(\Omega_{2}\right)$.

In accordance to [1], a state comprises of the program counter $n$ (which is a node in the CFG) and the valuation $\Omega$ of the variables in scope ${ }^{1}$. Unlike the conventional notion of a program state, a state in a Boolean program does not contain the content of the call stack.

We use the following notation to describe transition functions:

- $\Omega(e)$ denotes the evaluation of the expression $e$ according to the valuation $\Omega$ of the variables in $e$. Expressions and their evaluation are defined the usual

\footnotetext{
${ }^{1}$ A state corresponds to a head of a configuration in a pushdown system [11].
} 
way. Furthermore, we allow expressions to range over variables in two different states $\left\langle n_{1}, \Omega_{1}\right\rangle$ and $\left\langle n_{2}, \Omega_{2}\right\rangle$. We refer to the state before the execution of a transition function as current state, and to the state afterwards as next state.

- We use primed versions of the variables to distinguish variables that refer to the next state from the variables in the current state. $\left(\Omega_{1}, \Omega_{2}\right)(e)$ denotes the evaluation of such an expression $e$.

- Expressions may also contain non-deterministic choice. While non-deterministic values are traditionally represented by "*", we use a set of non-deterministic choice variables $\iota_{1}, \ldots \iota_{k}$ instead. We use $\xi$ to denote the set of these variables.

In the given setting, only the topmost element of the stack has an immediate impact on the execution of a transition. Therefore, the outcome of a call to a procedure is exclusively determined by the values of the global variables and the actual parameters at the call site. Consequently, each actual call to a procedure pr can be summarized by a pair of states $\left\langle n_{i}, \Omega_{i}\right\rangle,\left\langle n_{o}, \Omega_{o}\right\rangle$, where $n_{i}$ denotes the entry node of the control flow graph of $\mathbf{p r}$, and $n_{o}$ denotes the corresponding exit node. We use $\Sigma(\mathbf{p r})$ to denote the set of these pairs for a procedure pr. Furthermore, we assume in this section that $\Sigma(\mathbf{p r})$ contains the entries for all reachable call contexts. Clearly, $\Sigma(\mathbf{p r})$ is finite.

For a given entry state, the corresponding exit states ${ }^{2}$ are determined by the transition functions of the control flow graph of pr. The transition functions then again are determined by the statements corresponding to the nodes of the control flow graph. We distinguish following statements:

- The skip statement does not modify the variables, but increments the program counter by one.

- The goto $\ell_{1}, \ldots, \ell_{m}$ statement non-deterministically changes the program counter to one of the program locations $\ell_{1}, \ldots, \ell_{m}$ provided as argument. The valuation of the variables does not change.

- The assume $e$ statement increases the program counter by one iff the condition $e$ evaluates to true in the current state. Otherwise, the assume statement has no successor states, i.e., terminates the program.

- The constrained assignment statement $x_{1}, \ldots, x_{k}:=e_{1}, \ldots, e_{k}$ constrain $e$ assigns the values of the expressions $e_{1}, \ldots, e_{k}$ to the variables $x_{1}, \ldots, x_{k}$. The expressions are evaluated in the current state and may contain a nondeterministic choice variables. The constraint $e$ is a predicate that ranges over the variables of the current and the next state. It is evaluated in both states, and the statement has no successor state if $e$ does not hold.

- The return statement corresponds to the exit node of the control flow graph of pr. Whenever it is reached, the current state determines the exit valuation of the corresponding summary. We assume without loss of generality that all return values are passed to the caller via global variables, i.e., return has no parameters. Therefore, the variables are not modified. The program counter

\footnotetext{
${ }^{2}$ The use of non-determinism may result in more than one exit valuation.
} 
of the successor statement is determined by the caller of the corresponding procedure pr.

- The call $\operatorname{pr}\left(e_{1}, \ldots, e_{k}\right)$ modifies the global variables according to an applicable summary $\left\langle n_{i}, \Omega_{i}\right\rangle,\left\langle n_{o}, \Omega_{o}\right\rangle$ in $\Sigma(\mathbf{p r})$. A summary is applicable if a) $\Omega_{i}$ agrees with the current state on the global variables, and b) the evaluation of $e_{1}, \ldots, e_{k}$ matches the corresponding actual parameters in $\Omega_{i}$. (The calling context determines the entry valuation of a summary.)

Then, the call to $\boldsymbol{p r}\left(e_{1}, \ldots, e_{k}\right)$ modifies the global variables according to $\Omega_{o}$. If more than one summary is applicable, one summary is chosen nondeterministically (analogously to the goto statement).

In the case that an applicable summary exists, the call sets the program counter to the statement that succeeds the call. Otherwise, the statement succeeding the call is never reached.

The statements skip, assume, the constrained assignment, and procedure calls have a single successor node in the control flow graph (according to the structure of the program). The return statement has no successor in the control flow graph, since the program location that succeeds a return statement cannot be determined statically. Goto statements may have more than one successor. Conditional statements like if-then-else or while loops can be modeled using a combination of the goto and assume statements.

The recursive nature of the definition of the semantics indicates that the set of reachable states of a Boolean program can be obtained by means of a fixpoint computation. Several algorithms that compute the least fix-point of $\Sigma(\mathbf{p r})$ in order to determine the set of reachable states have been proposed $[1,10,11]$.

We present a QBF-based algorithm to compute the least fix-point for $\Sigma(\mathbf{p r})$ in the following section, and propose to use a SAT-based over-approximation of this fix-point in Section 4.

\section{Summarization using QBF}

In this section we describe how we compute the least fix-point of $\Sigma(\mathbf{p r})$ using forward symbolic execution and QBF-based fix-point detection.

The valuation of a symbolic state is represented in terms of a Boolean formula over non-deterministic choice variables $\iota_{1}, \ldots, \iota_{k}$, i.e., we use a parametric representation. Boolean formulas are defined the usual way.

Definition 5 (Symbolic State). A symbolic state is a triple $\langle n, \omega, \gamma\rangle$. The first component $n$ identifies the node in the control flow graph and is represented explicitly. The second component $\omega$ maps the variables $V$ of the Boolean program to formulas, representing a set of valuations for the variables in the symbolic state. Finally, $\gamma$ is a Boolean formula over the non-deterministic choice variables and represents the guard of the state. An unsatisfiable guard indicates that there is no concrete state represented by $\langle n, \omega, \gamma\rangle[8]$. 


\begin{tabular}{c|c|c} 
Instruction & $\gamma_{2}$ & $\omega_{2}$ \\
\hline \hline skip & $\gamma_{2}=\gamma_{1}$ & $\omega_{2}=\omega_{1}$ \\
\hline return & $\gamma_{2}=\gamma_{1}$ & $\omega_{2}=\omega_{1}$ \\
\hline goto $\ell_{1}, \ldots \ell_{k}$ & $\gamma_{2}=\gamma_{1}$ & $\omega_{2}=\omega_{1}$ \\
\hline assume $e$ & $\gamma_{2}=\left(\gamma_{1} \wedge \omega_{1}(e)\right)$ & $\omega_{2}=\omega_{1}$ \\
\hline $\begin{array}{c}x_{1}, \ldots, x_{k}:=e_{1}, \ldots, e_{k} \\
\text { constrain } e\end{array}$ & $\gamma_{2}=\left(\gamma_{1} \wedge\left(\omega_{1}, \omega_{2}\right)(e)\right)$ & $\omega_{2}=\left(\omega_{1}\left[x_{1} / \omega_{1}\left(e_{1}\right)\right] \ldots\left[x_{k} / \omega_{1}\left(e_{k}\right)\right]\right)$ \\
\hline
\end{tabular}

Table 1. Conditions on the symbolic transitions $\left\langle n_{1}, \gamma_{1}, \omega_{1}\right\rangle \rightarrow\left\langle n_{2}, \gamma_{2}, \omega_{2}\right\rangle$ for the statements skip, goto, assume, and the constrained assignment

Each symbolic state $\langle n, \omega, \gamma\rangle$ represents the set of explicit states

$$
\{\langle n, \Omega\rangle \mid \exists \xi \cdot \xi(\gamma) \wedge \forall v \in V \cdot \Omega(v)=\xi(\omega(v))\}
$$

The symbolic state $\left\langle\ell_{10},\left(\iota_{1} \vee \iota_{2}\right),\left\{a \mapsto \iota_{1}, b \mapsto\left(\neg \iota_{1} \wedge \iota_{2}\right)\right\}\right\rangle$, for instance, represents the explicit states $\left\langle\ell_{10},\{a \mapsto 0, b \mapsto 1\}\right\rangle$ and $\left\langle\ell_{10},\{a \mapsto 1, b \mapsto 0\}\right\rangle$. The valuation $\left\langle\iota_{1}, \iota_{2}\right\rangle=\langle 0,0\rangle$ is ruled out by the guard, and the valuations $\left\langle\iota_{1}, \iota_{2}\right\rangle=\langle 1,0\rangle$ and $\left\langle\iota_{1}, \iota_{2}\right\rangle=\langle 1,1\rangle$ yield the same explicit state.

Before we proceed to introduce our symbolic representation of summaries, we define the transition relations for the statements skip, goto, assume, and the constrained assignment. The conditions in Table 1 are equivalent to those presented in [8] (except for the return statement). We use the following notation: We write $\omega[x / e]$ for the mapping that maps $x$ to the formula $e$, while it agrees with the mapping $\omega$ on all other variables. Analogously to $\Omega(e)$ in Section 2, we use $\omega(e)$ to denote the evaluation of the expression $e$ according to the mapping $\omega$.

The program locations are omitted in Table 1, since they change according to the rules presented in Section 2. The components $\gamma$ and $\omega$ are modified as follows:

- In case of a skip, return, or goto statement, $\gamma$ as well as $\omega$ do not change.

- Conditions contributed by assume statements are instantiated according to $\omega_{1}$ and conjoined with the guard $\gamma_{1}$. The symbolic execution terminates if $\gamma_{2}$ is unsatisfiable.

- A constrained assignment updates the mapping $\omega_{2}$ accordingly. If a constraining condition is present, it is instantiated using $\omega_{1}$ and $\omega_{2}$, and conjoined with $\gamma_{1}$.

Obviously, an actual symbolic transition can be characterized by a pair of symbolic states $\left\langle n_{1}, \gamma_{1}, \omega_{1}\right\rangle,\left\langle n_{2}, \gamma_{2}, \omega_{2}\right\rangle$. The first state represents the concrete set of states before the transition, and the second the corresponding concrete states afterwards. By construction (see Table 1), the components $\gamma_{1}, \gamma_{2}, \omega_{1}$, and $\omega_{2}$ share sub-formulas. Therefore, given one of the concrete states $\left\langle n_{1}, \Omega_{1}\right\rangle \in$ $\left\langle n_{1}, \gamma_{1}, \omega_{1}\right\rangle$, we can obtain the states that are reachable from $\left\langle n_{1}, \Omega_{1}\right\rangle$ via this transition by constraining the state $\left\langle n_{2}, \gamma_{2}, \omega_{2}\right\rangle$ : 


$$
\left\langle n_{2}, \quad\left(\gamma_{2} \wedge \bigwedge_{v \in V} \omega_{1}(v)=\Omega_{1}(v)\right), \omega_{2}\right\rangle
$$

Continuing our example, we construct the symbolic successor for the state $\left\langle\ell_{10},\left(\iota_{1} \vee \iota_{2}\right),\left\{a \mapsto \iota_{1}, b \mapsto\left(\neg \iota_{1} \wedge \iota_{2}\right)\right\}\right\rangle$ and the following statement:

$$
a:=\neg a \text { constrain }\left(\neg b \vee a^{\prime}\right)
$$

According to Table 1, we obtain $\omega_{2}=\left\{a \mapsto \neg \iota_{1}, b \mapsto\left(\neg \iota_{1} \wedge \iota_{2}\right)\right\}$ and $\gamma_{2}=\left(\iota_{1} \vee\right.$ $\left.\iota_{2}\right) \wedge\left(\iota_{1} \vee \neg \iota_{2} \vee \neg \iota_{1}\right) \equiv\left(\iota_{1} \vee \iota_{2}\right)$. We compute the successor states for the concrete state $\left\langle\ell_{10},\{a \mapsto 0, b \mapsto 1\}\right\rangle$ by adding the constraint $\iota_{1}=0 \wedge\left(\neg \iota_{1} \wedge \iota_{2}\right)=1$ to $\gamma_{2}$, ruling out the successor state $\left\langle\ell_{11},\{a \mapsto 0, b \mapsto 1\}\right\rangle$ and leaving us with $\left\langle\ell_{11},\{a \mapsto 1, b \mapsto 0\}\right\rangle$.

Equation 3 can be extended in order to constrain the transition with a symbolic state $\left\langle n_{1}, \gamma_{0}, \omega_{0}\right\rangle$ :

$$
\left\langle n_{2},\left(\gamma_{2} \wedge \gamma_{0} \wedge \bigwedge_{v \in V} \omega_{1}(v)=\omega_{0}(v)\right), \omega_{2}\right\rangle
$$

In (4), we assume that the non-deterministic choice variables in $\left\langle n_{1}, \gamma_{0}, \omega_{0}\right\rangle$ differ from those in $\left\langle n_{1}, \gamma_{1}, \omega_{1}\right\rangle$ and $\left\langle n_{2}, \gamma_{2}, \omega_{2}\right\rangle$. This can always be achieved by renaming.

The representation of transitions by means of two symbolic states is not restricted to single transitions, but can be extended to sequences of transitions in the natural way. This representation enables the summarization of compound transitions, and is similar to the concept of path edges [1].

Definition 6 (Path edges and summary edges). A pair of symbolic states $\left\langle n_{i}, \gamma_{i}, \omega_{i}\right\rangle,\left\langle n_{o}, \gamma_{o}, \omega_{o}\right\rangle$ is a path edge of procedure $\mathbf{p r}$ iff

$-n_{i}$ is the entry node of $\mathbf{p r}$.

- $\left\langle n_{i}, \gamma_{i}, \omega_{i}\right\rangle$ is reachable from an initial state of the Boolean program.

- $\left\langle n_{o}, \gamma_{o}, \omega_{o}\right\rangle$ is reachable from $\left\langle n_{i}, \gamma_{i}, \omega_{i}\right\rangle$ by a sequence of statements that does not contain the return statement of pr (unless $n_{o}$ happens to be the corresponding exit node).

In particular, we can use this representation to store summaries for procedure calls. We define a summary edge of pr to be a path edge $\left\langle n_{i}, \gamma_{i}, \omega_{i}\right\rangle,\left\langle n_{o}, \gamma_{o}, \omega_{o}\right\rangle$, for which we require that $n_{i}$ corresponds to the entry node, and $n_{o}$ corresponds to the exit node of $\mathbf{p r}$.

Using Definition 6 and Equation 4, we can give a symbolic transition function for the procedure call statement. Assume that we encounter a procedure call $\operatorname{pr}\left(e_{1}, \ldots, e_{k}\right)$ and the current state is $\left\langle n_{c}, \gamma_{c}, \omega_{c}\right\rangle$. Let $\Sigma_{s}(\mathbf{p r})$ be the set of symbolic summaries for the procedure pr. We use $g_{1}, \ldots, g_{m}$ to denote the global variables of the Boolean program, $l_{1}, \ldots, l_{j}$ to denote the local variables of the 
calling context, and $f_{1}, \ldots, f_{k}$ to denote the formal parameters of the procedure pr.

A summary edge $\left\langle n_{i}, \gamma_{i}, \omega_{i}\right\rangle,\left\langle n_{o}, \gamma_{o}, \omega_{o}\right\rangle \in \Sigma_{s}(\mathbf{p r})$ is applicable to the calling context $\left\langle n_{c}, \gamma_{c}, \omega_{c}\right\rangle$ iff

1. for all reachable valuations of $g_{1}, \ldots, g_{m}, e_{1}, \ldots, e_{k}$ in $\left\langle n_{c}, \gamma_{c}, \omega_{c}\right\rangle$ there exists a matching valuation $g_{1}, \ldots, g_{m}, f_{1}, \ldots, f_{k}$ in $\left\langle n_{i}, \gamma_{i}, \omega_{i}\right\rangle$, and

2. $\gamma_{o}$ is still satisfiable when the global and formal variables are restricted to the global variables and parameter expressions of $\gamma_{c}$ and $\omega_{c}$ according to Equation 4.

The universal quantification in the first condition requires us to use a QBF instance to decide applicability:

$$
\forall \xi_{c} \cdot \gamma_{c} \Rightarrow \exists \xi_{o} . \bigwedge_{s \in\{1 . . m\}} w_{i}\left(g_{s}\right)=w_{c}\left(g_{s}\right) \wedge \bigwedge_{t \in\{1 . . k\}} w_{i}\left(f_{t}\right)=w_{c}\left(e_{t}\right) \wedge \gamma_{o}
$$

We use $\xi_{c}$ to denote the set of non-deterministic choice variables in $\gamma_{c}$ and $\omega_{c}$, and $\xi_{o}$ for the set of remaining non-deterministic choice variables. Again, we assume that the sets $\xi_{c}$ and $\xi_{o}$ are disjoint.

Assuming that (5) holds, we can restrict the symbolic state $\left\langle n_{o}, \gamma_{o}, \omega_{o}\right\rangle$ to the states reachable from the calling context $\left\langle n_{c}, \gamma_{c}, \omega_{c}\right\rangle$ analogously to (4). By applying the summary, we obtain a new symbolic state $\left\langle n_{r}, \gamma_{r}, \omega_{r}\right\rangle$ (the state after the return statement) with

$$
\gamma_{r}:=\gamma_{o} \wedge \gamma_{c} \wedge \bigwedge_{s \in\{1 . . m\}} w_{i}\left(g_{s}\right)=w_{c}\left(g_{s}\right) \wedge \bigwedge_{t \in\{1 . . k\}} w_{i}\left(f_{t}\right)=w_{c}\left(e_{t}\right)
$$

and

$$
\omega_{r}\left(g_{1}\right)=\omega_{o}\left(g_{1}\right), \ldots, \omega_{r}\left(g_{m}\right)=\omega_{o}\left(g_{m}\right), \omega_{r}\left(l_{1}\right)=\omega_{c}\left(l_{1}\right), \ldots, \omega_{r}\left(l_{j}\right)=\omega_{c}\left(l_{j}\right)
$$

Now consider the case that (5) does not hold, i.e., $\Sigma_{s}(\mathbf{p r})$ contains no applicable summary. In that case, a new summary edge must to be computed for the calling context $\left\langle n_{c}, \gamma_{c}, \omega_{c}\right\rangle$. For this purpose, we construct a new symbolic state $\left\langle n_{i}, \gamma_{i}, \omega_{i}\right\rangle$ which agrees with $\left\langle n_{c}, \gamma_{c}, \omega_{c}\right\rangle$ on the global variables, and as$\operatorname{sign} e_{1}, \ldots, e_{k}$ to the formal parameters (using same transition function as the assignment statement). The symbolic state $\left\langle n_{i}, \gamma_{i}, \omega_{i}\right\rangle$ serves as entry node for a new path edge, and may eventually yield a new summary edge.

Fix-point Detection In order to determine the least fix-point of $\Sigma_{s}(\mathbf{p r})$, our reachability checking algorithm maintains a set $\mathcal{P}$ of path edges that have been constructed so far. Our algorithm is similar to the BDD-based model checking algorithm presented in [1]. However, unlike a BDD-based representation of path edges, our representation is not canonical. The price we pay for being able to 


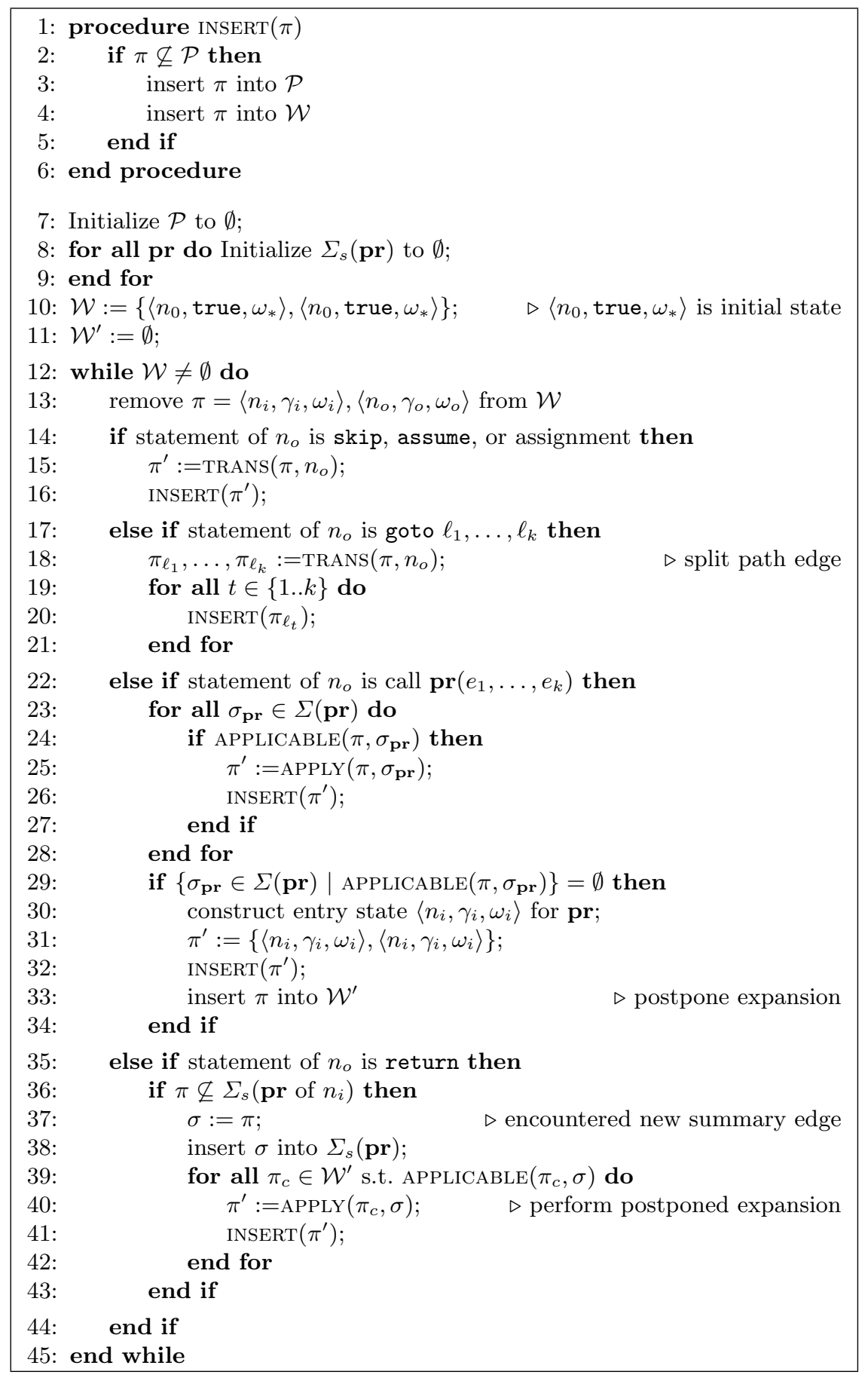

Fig. 1. The SAT based model checking algorithm 
apply transition functions efficiently is that we need to solve a QBF instance in order to determine whether a path edge is already an element of $\mathcal{P}$.

Let $V$ be the variables in scope in procedure pr. Given two path edges $\left\langle n_{i}, \gamma_{i 1}, \omega_{i 1}\right\rangle,\left\langle n_{o}, \gamma_{o 1}, \omega_{o 1}\right\rangle$ and $\left\langle n_{i}, \gamma_{i 2}, \omega_{i 2}\right\rangle,\left\langle n_{o}, \gamma_{o 2}, \omega_{o 2}\right\rangle$, the latter is at least as general as the former iff

$$
\forall \xi_{1} \cdot \gamma_{o 1} \Rightarrow \exists \xi_{2} \cdot \gamma_{o 2} \wedge \bigwedge_{v \in V} \omega_{i 1}(v)=\omega_{i 2}(v) \wedge \omega_{o 1}(v)=\omega_{o 2}(v)
$$

where $\xi_{1}$ refers to the non-deterministic choice variables of the first path edge, and $\xi_{2}$ to those of the second. Equation 8 holds iff the set of pairs of concrete states represented by the first path edge is a subset of the corresponding set represented by the second. In that case, a further expansion of the path edge $\left\langle n_{i}, \gamma_{i 1}, \omega_{i 1}\right\rangle,\left\langle n_{o}, \gamma_{o 1}, \omega_{o 1}\right\rangle$ does not yield any states that are not discovered by expanding the more general path edge.

The pseudo code of our QBF-based algorithm is presented in Figure 1. It resembles the model checking algorithm presented in [1], but uses SAT and QBF instead of BDDs. In line 10, we use $\omega_{*}$ to indicate that the state is initialized non-deterministically.

We use $\operatorname{Applicable}(\pi, \sigma)$ to denote the condition in Equation 5, where $\pi=$ $\left\langle n_{e}, \gamma_{e}, \omega_{e}\right\rangle,\left\langle n_{c}, \gamma_{c}, \omega_{c}\right\rangle$ is a path edge that provides the calling context, and $\sigma$ is a summary edge. Furthermore, $\operatorname{APPly}(\pi, \sigma)$ denotes the path edge that we obtain by applying the summary according to equations (6) and (7). The condition in Equation 8 is expressed by $\pi_{1} \subseteq \pi_{2}$ and holds if the path edge $\pi_{1}$ is subsumed by $\pi_{2}$. Finally, we use $\operatorname{TRANS}(\pi, n)$ to denote the application of the transition function of a node $n$ as listed in Table 1 .

The algorithm maintains a work-list $\mathcal{W}$ in which all path-edges are stored that are currently explored are stored. Each path edge of this work-list is expanded according to the transition functions described above, until either the guard becomes unsatisfiable or the resulting path edge is already in $\mathcal{P}$. For convenience, we define a procedure insert $(\pi)$, which we use to insert a path edge into the work-list, unless it is already contained in $\mathcal{P}$.

In line 14, the transition functions presented in Table 1 are applied. Whenever the algorithm encounters a goto statement, the current path edge is split (see line 17).

Procedure calls are handled in line 22. Matching summary edges are applied immediately. However, if there is no applicable summary edge, we construct an entry state for the called procedure and add a corresponding path edge to the work-list $\mathcal{W}$. Furthermore, we store the current path $\sigma$ edge in $\mathcal{W}^{\prime}$, which is examined whenever we add a new summary to $\Sigma(\mathbf{p r})$. Thus, we guarantee that any summary of pr that is eventually generated is applied to also $\sigma$ (see line 39).

Path merging Splitting the path edge in line 17 of our algorithm in Figure 1 may lead to an explosion of the of the number of path edges. Therefore, we merge path edges in our work-list $\mathcal{W}$ whenever possible. Two path edges $\left\langle n_{i 1}, \gamma_{i 1}, \omega_{i 1}\right\rangle,\left\langle n_{o 1}, \gamma_{o 1}, \omega_{o 1}\right\rangle$ and $\left\langle n_{i 2}, \gamma_{i 2}, \omega_{i 2}\right\rangle,\left\langle n_{o 2}, \gamma_{o 2}, \omega_{o 2}\right\rangle$ can be merged if 
$\left\langle n_{i 1}, n_{o 1}\right\rangle$ and $\left\langle n_{i 2}, n_{o 2}\right\rangle$ coincide. In that case, we construct a new path edge

$\left\langle n_{i 1}, \gamma_{i}, \omega_{i}\right\rangle,\left\langle n_{o} 1, \gamma_{o}, \omega_{o}\right\rangle$ such following conditions hold for $\gamma_{i}$ and $\omega_{i}$ :

$-\gamma_{i}=\left(\gamma_{i 1} \wedge \bigwedge_{v \in V} \omega_{i}(v)=\omega_{i 1}(v)\right) \vee\left(\gamma_{i 2} \wedge \bigwedge_{v \in V} \omega_{i}(v)=\omega_{i 2}(v)\right)$

- for all $v \in V, \omega_{i}(v)=\omega_{i 1}(v) \vee \omega_{i 2}(v)$

Analogously, we construct similar conditions for $\gamma_{o}$ and $\omega_{o}$ and name the procedure that merges a set of path edges $\Pi \operatorname{MERGE}(\Pi)$. We deploy a heuristic that postpones the application of certain transitions (e.g. at join nodes in the CFG) in order to increase the number of path edges in $\mathcal{W}$ that can be merged. A similar approach is used by the model checker DizzY [20], but not in combination with summarization.

\section{Universal Summaries}

Solving the QBF instances is the primary performance bottleneck of the algorithm presented in the previous section. The majority of the QBF instances is generated by the fix-point detection algorithm (see Equation 8). These QBF instances cannot be avoided if we want to compute the least fix-point of $\Sigma_{s}(\mathbf{p r})$. However, if we settle for an over-approximation of this fix-point, we can reduce the number of calls to the QBF solver significantly.

The set of path edges consisting of all sequences of transitions of a procedure pr up to its reachability recurrence diameter (see Def. 3) is such an overapproximation. The definition of the reachability recurrence diameter requires adaption to be applicable to procedures of Boolean programs:

- The reachability recurrence diameter is only defined for finite state system and therefore not applicable to recursive procedure calls.

- The transition function $T$ in Definition 1 is a compound, synchronous transition function that modifies all state variables in each step. A Boolean program is a disjunctive partitioning of local transitions, and the advantage of locality is lost if we treat it as a compound transition function.

We address these issues as follows:

- We replace the procedure calls in pr by a non-deterministic assignment to all global variables that are potentially changed by the callee. The set of these variables can be obtained by static analysis. The resulting procedure $\mathbf{p r}^{*}$ is an over-approximation of the behavior of pr.

- We still split the path edges, but perform aggressive merging, i.e., we merge at every join node in the CFG. Thus, instead of unwinding the entire compound transition function, each cycle in the CFG is unwound separately. This corresponds to the loop unrolling algorithm used in the CBMC tool [24].

The algorithm in Figure 2 performs aggressive merging by making sure that no path edge can proceed beyond a join node unless all other path edges in the work list have "caught up". We achieve this by assigning a priority to each node 


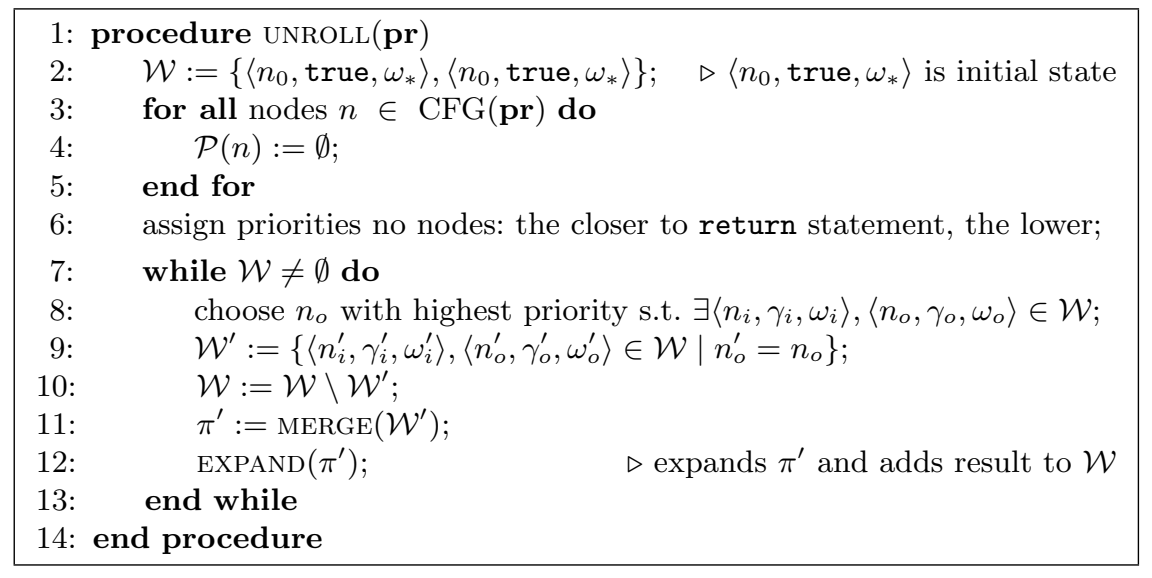

Fig. 2. Expanding and merging path edges at every join node

in the control flow graph. The priority depends on the distance to the exit node: Nodes closer to the return statement have a lower priority, and the exit node itself has the lowest priority.

Note that this algorithm fails to proceed beyond a join node of a cycle of the CFG if the procedure ExPAND (see call in line 12) perpetually generates new path edges for this cycle. We prevent this by restricting the path edges generated by EXPAND to the reachability recurrence diameter of pr:

Definition 7 (Reachability Recurrence Diameter of procedure). The reachability recurrence diameter $\operatorname{rrd}(\mathbf{p r})$ of a Boolean procedure $\mathbf{p r}$ is the longest sequence of concrete transitions in $\mathrm{pr}^{*}$ such that no state $\langle n, \Omega\rangle$ of the procedure is visited twice.

Given a set of symbolic path edges $\left\langle n_{i}, \gamma_{i}, \omega_{i}\right\rangle,\left\langle n_{o}, \gamma_{o, j}, \omega_{o, j}\right\rangle, j \in\{1 . . n\}$ for a node $n_{o}$ in procedure pr*, we can construct a formula (similar to Equation 2) that is satisfiable iff there are $n$ distinct states such that each path edge represents one of them:

$$
\exists \xi \cdot \bigwedge_{j=0}^{n-1} \bigwedge_{k=j+1}^{n} \gamma_{o, j} \wedge \gamma_{o, k} \wedge \bigwedge_{v \in V} \omega_{o, j}(v) \neq \omega_{o, k}(v)
$$

If we keep a record of all path edges that reach a node $n$ (using a set $\mathcal{P}(n)$ ), we can use Equation 9 to check whether there still exists a sequence of concrete transitions that visits no state at $n$ twice. We use $\operatorname{RECURRING}(\mathcal{P}(n))$ to denote Equation 9.

The pseudo-code for the procedure EXPAND is presented in Figure 3. The algorithm checks at each program location whether the reachability recurrence diameter is exceeded. The path edge of the current step is added to $\mathcal{P}(n)$ unless $\operatorname{RECURRING}(\mathcal{P} \cup\{\pi\})$ holds (we implicitly assume that path edges with unsatisfiable guards are dropped, too). 


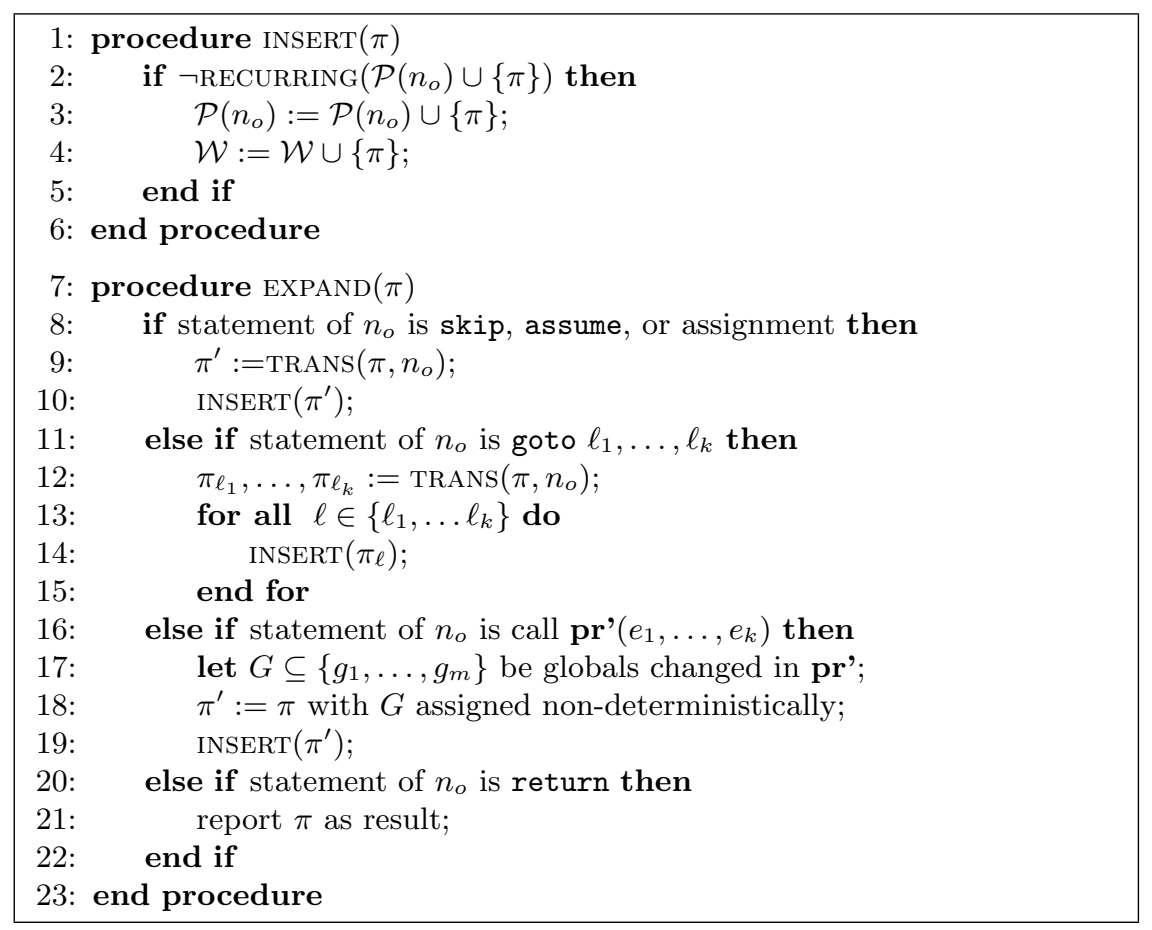

Fig. 3. Unrolling transitions of a Boolean procedure until states repeat

Whenever the algorithm encounters a call to a procedure pr', it replaces the transition by a non-deterministic assignment to the globals that are potentially changed by pr' (see line 18). Finally, we return the entirely merged path edge $\pi$ that reaches the exit node of the CFG.

Again, we use $\left\langle n_{0}\right.$, true, $\left.\omega_{*}\right\rangle$ to denote the non-deterministically initialized state (see line 2 in Figure 2), i.e., we do not restrict the calling context. Therefore, our algorithm generates a path edge that contains all sequences of transitions that do not visit a state twice for an arbitrary calling context.

We use this observation to justify our initial claim: The path edge $\pi$ obtained by computing UNROLL(pr) over-approximates $\Sigma_{s}(\mathbf{p r})$. We call this $\pi$ a universal summary.

Definition 8 (Universal summary). The universal summary $\Sigma_{u}(\mathbf{p r})$ of $a$ procedure $\mathbf{p r}$ is the path edge that we obtain by merging the path edges of all sequences of transitions (the initial state being an unconstrained calling context) of $\mathbf{p r}^{*}$ up to the reachability recurrence diameter of $\mathbf{p r}^{*}$, i.e., $\Sigma_{u}(\mathbf{p r}):=$ $\operatorname{UNROLL}(\mathbf{p r})$.

The universal summary of $\mathbf{p r}$ does not only over-approximate $\Sigma_{s}(\mathbf{p r})$, but also the set of feasible transition sequences. These spurious execution traces are 
eliminated when $\Sigma_{u}(\mathbf{p r})$ is applied at all call sites of $\mathbf{p r}$ according to equations (6) and (7).

However, in case of a cyclic dependency between procedures (i.e., in case of recursion or mutual recursion) the universal summaries cannot be applied. Therefore, we compute the universal summaries of all non-recursive procedures of a Boolean program, and use the algorithm in Figure 1 to handle the remaining recursive procedure calls.

\section{Benchmarks}

We used Slam [9] and Terminator [25] to generate eight Boolean programs from Windows device drivers. We compare the performance of our implementation on these examples to the model checker BEBOP, which is part of Microsoft Research's SLAM/SDV toolkit [9] (see Table 2). In addition, we compare the effect of summarization with universal summaries and entirely QBF-based summarization. The column labeled "violation" indicates whether the reachability property we are checking for can be violated or not.

\begin{tabular}{|c|c|c|c|c|c|}
\hline Benchmark & \#vars & BEBOP & QBF-summaries & univ. summ. & violation \\
\hline \hline SLAM adddevice & 643 & $4 \mathrm{~m} 37.4 \mathrm{~s}$ & $0 \mathrm{~m} 15.9 \mathrm{~s}$ & $0 \mathrm{~m} 15.7 \mathrm{~s}$ & yes \\
\hline SLAM nulldevice & 856 & $4 \mathrm{~m} 34.0 \mathrm{~s}$ & $0 \mathrm{~m} 7.1 \mathrm{~s}$ & $0 \mathrm{~m} 5.8 \mathrm{~s}$ & yes \\
\hline SLAM pendedcompletedreq & 86 & $0 \mathrm{~m} 30.9 \mathrm{~s}$ & $0 \mathrm{~m} 2.7 \mathrm{~s}$ & $0 \mathrm{~m} 3.2 \mathrm{~s}$ & yes \\
\hline SLAM targetrelationneedsref & 37 & $0 \mathrm{~m} 0.4 \mathrm{~s}$ & $0 \mathrm{~m} 1.8 \mathrm{~s}$ & $0 \mathrm{~m} 1.8 \mathrm{~s}$ & no \\
\hline SLAM markirppending & 11 & $0 \mathrm{~m} 0.4 \mathrm{~s}$ & $0 \mathrm{~m} 2.4 \mathrm{~s}$ & $0 \mathrm{~m} 2.3 \mathrm{~s}$ & no \\
\hline SLAM wmiforward & 15 & $0 \mathrm{~m} 0.7 \mathrm{~s}$ & $0 \mathrm{~m} 0.5 \mathrm{~s}$ & $0 \mathrm{~m} 0.5 \mathrm{~s}$ & no \\
\hline TERMINATOR 1 & 74 & timeout & $1 \mathrm{~m} 55.9 \mathrm{~s}$ & $1 \mathrm{~m} 55.9 \mathrm{~s}$ & yes \\
\hline TERMINATOR 2 & 60 & $88 \mathrm{~m} 22.6 \mathrm{~s}$ & timeout & timeout & yes \\
\hline
\end{tabular}

Table 2. Comparison of performance of BEBOP, QBF-based summarization, and universal summaries (timout: $>2 h$ )

The algorithm that uses universal summaries deploys a heuristic that switches back to QBF-summaries for procedures with universal summaries that are larger than a certain threshold. This typically happens if the procedure contains a lot of non-deterministic assignments.

The benchmarks are very incoherent, and we have yet to investigate why this is the case. In some situations (like the adddevice benchmark with 643 variables), our implementation is significantly faster than BEBOP. However, this cannot be generalized. Furthermore, the benchmarks show that it is not always advantageous to use universal summaries. The QBF-instances resulting from the combination of universal summaries and QBF-based summarization may become too large for the solver SkIzzo [26]. In the TERMinATOR 2 benchmark, this also happens without universal summaries.

We have a large number of small regression tests that indicate that BDDbased implementations are still faster for Boolean programs with a small number 
of variables. However, the reason for this may be that we did not profile and optimize our implementation, yet. We intend to make an updated set of benchmarks available as soon as we are able to explain the performance problems on small examples.

\section{Conclusion}

We present a SAT based model checking algorithm for Boolean programs that uses a QBF solver to compute the least fix-point of the set of summary edges of a procedure. Furthermore, we introduce the concept of universal summaries, an over-approximation of the summary edges our initial algorithm computes. By using universal summaries, we reduce the number of calls to the QBF solver significantly.

Our preliminary benchmarks do not allow us to conclude that our approach is in general superior than BDD based model checking. However, some of the results are very promising and indicate that it is worthwhile to further pursue the idea.

Future Work We need to spend more time testing and optimizing our implementation, and investigating why BЕBOP performs better on small programs.

\section{References}

1. Ball, T., Rajamani, S.K.: Bebop: A symbolic model checker for Boolean programs. In: Model Checking and Software Verification (SPIN). Volume 1885 of LNCS., Springer (2000) 113-130

2. Ball, T., Rajamani, S.: Boolean programs: A model and process for software analysis. Technical Report 2000-14, Microsoft Research (2000)

3. Graf, S., Saïdi, H.: Construction of abstract state graphs with PVS. In: Computer Aided Verification (CAV). Volume 1254 of LNCS., Springer (1997) 72-83

4. Büchi, J.R.: Regular canonical systems. Archive for Mathematical Logic 6 (1964) 91

5. Sharir, M., Pnueli, A.: Two approaches to interprocedural data dalow analysis. In: Program Flow Analysis: Theory and Applications. Prentice-Hall (1981) 189-233

6. Esparza, J., Hansel, D., Rossmanith, P., Schwoon, S.: Efficient algorithms for model checking pushdown systems. In: Computer Aided Verification (CAV). Volume 1855 of LNCS., Springer (2000) 232-247

7. Bryant, R.E.: Graph-based algorithms for Boolean function manipulation. IEEE Transactions on Computers 35 (1986) 677-691

8. Cook, B., Kroening, D., Sharygina, N.: Symbolic model checking for asynchronous Boolean programs. In: Model Checking and Software Verification (SPIN). Volume 3639 of LNCS., Springer (2005) 75-90

9. Ball, T., Cook, B., Levin, V., Rajamani, S.K.: SLAM and Static Driver Verifier: Technology transfer of formal methods inside Microsoft. In: Integrated Formal Methods. Volume 2999 of LNCS., Springer (2004) 
10. Finkel, A., Willems, B., Wolper, P.: A direct symbolic approach to model checking pushdown systems. ENTCS 9 (1997)

11. Schwoon, S.: Model-Checking Pushdown Systems. PhD thesis, Technische Universität München (2002)

12. Lal, A., Reps, T.: Improving pushdown system model checking. In: Computer Aided Verification (CAV). LNCS, Springer (2006) 343-357

13. Bouajjani, A., Esparza, J.: Rewriting models of Boolean programs. In: Rewriting Techniques and Applications (RTA). Volume 4098 of LNCS. (2006)

14. Bouajjani, A., Esparza, J., Touili, T.: A generic approach to the static analysis of concurrent programs with procedures. In: Principles of Programming Languages (POPL), ACM (2003) 62-73

15. Bouajjani, A., Touili, T.: On computing reachability sets of process rewrite systems. In: Rewriting Techniques and Applications (RTA). Volume 3467 of LNCS., Springer (2005) 484-499

16. Ramalingam, G.: Context-sensitive synchronization-sensitive analysis is undecid able. ACM Transactions on Programming Languages and Systems 22 (2000) 416430

17. Qadeer, S., Rehof, J.: Context-bounded model checking of concurrent software. In: Tools and Algorithms for the Construction and Analysis of Systems (TACAS). Volume 3440 of LNCS., Springer (2005) 93-103

18. Touili, T., Sighireanu, M.: Bounded communication reachability analysis of process rewrite systems with ordered parallelism. In: Verification of Infinite State Systems (INFINITY), Elsevier (2007) To appear.

19. Cook, B., Kroening, D., Sharygina, N.: Over-approximating Boolean programs with unbounded thread creation. In: Formal Methods in Computer-Aided Design (FMCAD), IEEE (2006) 53-59

20. Leino, K.R.M.: A SAT characterization of Boolean-program correctness. In: Model Checking and Software Verification (SPIN). Volume 2648., Springer (2003) 104-120

21. Biere, A., Cimatti, A., Clarke, E.M., Zhu, Y.: Symbolic model checking without BDDs. In: Tools and Algorithms for the Construction and Analysis of Systems (TACAS). Volume 1579 of LNCS., Springer (1999) 193-207

22. Kroening, D., Strichman, O.: Efficient computation of recurrence diameters. In: Verification, Model Checking and Abstract Interpretation (VMCAI). Volume 2575 of LNCS., Springer (2003) 298-309

23. Biere, A., Cimatti, A., Clarke, E.M., Strichman, O., Zhu, Y.: Bounded model checking. Advances in Computers 58 (2003) 118-149

24. Clarke, E., Kroening, D., Lerda, F.: A tool for checking ANSI-C programs. In: Tools and Algorithms for the Construction and Analysis of Systems (TACAS). Volume 2988 of LNCS., Springer (2004) 168-176

25. Cook, B., Podelski, A., Rybalchenko, A.: Terminator: Beyond safety. In: Computer Aided Verification (CAV). Volume 4144 of LNCS., Springer (2006)

26. Benedetti, M.: Evaluating QBFs via symbolic skolemization. In: Logic for Programming, Artificial Intelligence, and Reasoning. Volume 3452 of LNCS. Springer (2005) 285-300 